\title{
Ultrasonic measurement of gas flow using electrostatic transducers
}

\author{
I.J. O'Sullivan, W.M.D. Wright * \\ Department of Electrical and Electronic Engineering, University College Cork, College Road, Cork, Ireland
}

\begin{abstract}
Ultrasonic gas flowmeters typically use narrowband piezoelectric transducer arrangements for interrogating the flow of gas in a pipe. In this work, the suitability of broadband electrostatic transducers operating at frequencies of up to $1 \mathrm{MHz}$ for ultrasonic measurement of gas flow has been investigated. The transit time method of ultrasonic gas flow measurement was adopted and experiments were carried out using a laboratory test rig capable of producing a range of gas flowrates up to $17.5 \mathrm{~m} / \mathrm{s}$. The test rig also allowed easy interchange of different prototype flowmetering sections. Times of flight of ultrasonic waves interrogating the gas flow were measured using separate send/receive electrostatic transducer arrangements. Two flowmeter configurations were considered. The first interrogated the flow at $45^{\circ}$ in contra-propagating upstream and downstream directions. The second consisted of an upstream interrogation at $45^{\circ}$ to the gas flow and an interrogation made normal to the flow direction. $k$ factors correlating the fluid velocity along the ultrasonic path with the mean fluid velocity in the pipe were calculated using experimental ultrasonic data and anemometer measurements. All transducer configurations were numerically modelled using the computational fluid dynamics software package FLOTRAN ${ }^{\odot}$ (ANSYS Inc.). Theoretical gas flow velocities for both transducer arrangements were subsequently compared with experimental values and found to be in excellent agreement. A flow-dependent frequency shift of the received ultrasonic signals was also observed simultaneously with the transit time measurement. (c) 2002 Elsevier Science B.V. All rights reserved.
\end{abstract}

Keywords: Ultrasonic Gas Flow Measurement; Gas Flow; Electrostatic Transducers; FLOTRAN ${ }^{\odot}$ (ANSYS Inc.) modelling

\section{Introduction}

Quantification of fluid flow may be carried out using any of the following methods: (i) a mass flowrate measurement using angular momentum or 'Wheatstone Bridge' meters [1], (ii) a point velocity measurement using a Pitot tube, vane or hot wire anemometer [2-4], and (iii) a mean volumetric flowrate measurement using a differential pressure meter or rotating mechanical meter [5]. These flowmeters are all well characterised and readily available. However most cause an unsatisfactory obstruction to flow and/or associated pressure drop.

An alternative volumetric flowrate measurement may be made using an ultrasonic flowmeter, which offers little or no obstruction to fluid flow. This type of device is capable of operating under a wide range of conditions including unsteady flow [6], and in single and two phase

\footnotetext{
* Corresponding author. Tel.: +353-21-4902213; fax: +353-214271698.

E-mail address: bill.wright@ucc.ie (W.M.D. Wright).
}

media [7]. Ultrasonic flow measurements up to $30 \mathrm{~m} / \mathrm{s}$ in liquids [8] and beyond Mach 1 for gases have been reported [9]. Conduit sizes may vary from millimetres to metres in diameter [8], and temperatures from cryogenic $\left(-40^{\circ} \mathrm{C}\right)$ up to $250^{\circ} \mathrm{C}$ and pressures up to 100 bar can be tolerated $[9,10]$. Response times of the order $1 \mathrm{~ms}$ can be achieved [9], and transducer installation configurations such as clamp-on, insert probe, spool piece, and hot tapped ports are common [7].

Ultrasonic flowmeters for liquids are commonplace, and they generally utilize piezoelectric transducers to generate and detect the ultrasound. However, difficulties arise when attempting to measure gas flowrates ultrasonically, due mainly to the mismatch in specific acoustic impedance between most piezoelectric materials and gases. Piezoelectric transducers are available for use in gas flowmeters but they usually have a high acoustic impedance, and a narrow bandwidth as a resonant quarter wave impedance matching layer is necessary.

A transducer more suitable for generating and receiving ultrasound in gases is based on an electrostatic principle [11-15], and is shown schematically in Fig. 1. It typically consists of two electrodes, one a 


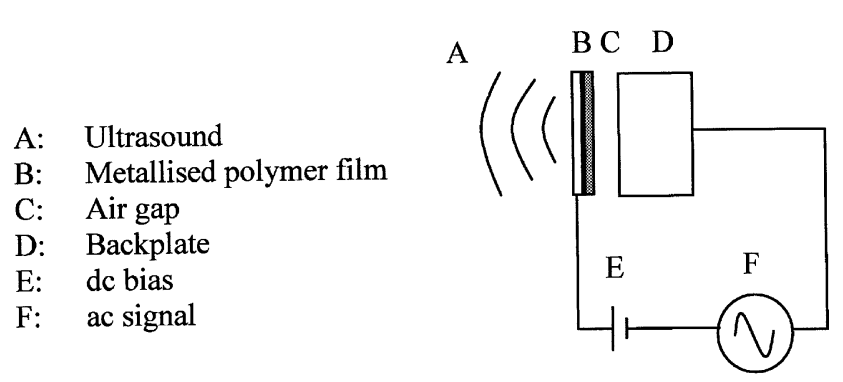

Fig. 1. Schematic diagram of a typical electrostatic transducer.

metallised flexible polymer membrane, and the other a rigid contoured conducting back plate, to which a dc bias voltage is applied. The bias voltage builds up charge between the two electrodes, and the flexible membrane can be made to vibrate under the application of an ac stimulus. This type of device is extremely sensitive, demonstrates good bandwidth characteristics due to its nonresonant nature, and can generate and detect ultrasound in gases at frequencies in excess of $1 \mathrm{MHz}$ [12]. The membrane thickness, bias voltage and the surface properties of the back plate influence the beam profile, frequency response and sensitivity of the transducer [11-15]. The use of electrostatic transducers in gas flowmetry has received little attention to date although previous work has investigated their use in tomographic reconstruction of flow fields in gases [16].

There are four main techniques of employing ultrasonic fluid flow measurement: Doppler [17], correlation [18], fluid oscillatory [19] and transit time [7]. The objective of the current study is to investigate the application of high frequency broadband electrostatic ultrasonic gas-coupled transducers in transit time ultrasonic flowmeters.

\section{Theory of transit time ultrasonic flowmeters}

A typical transit time ultrasonic flowmeter is shown schematically in Fig. 2(a). It measures the difference between the time of flight of ultrasonic waves transmitted upstream and downstream in the flowing medium. The fluid velocity $v$ in $\mathrm{m} / \mathrm{s}$ along the ultrasonic path in a pipe of diameter $d$ in metres at an angle $\theta$ to the flow direction is given by [7]:

$v=\frac{d \Delta t}{2 t_{1} t_{2} \sin \theta \cos \theta}$

where $t_{1}$ is the time of flight in seconds for upstream path $L_{\mathrm{U}}, t_{2}$ is the time of flight in seconds for downstream path $L_{\mathrm{D}}$, and $\Delta t$ is the difference between $t_{1}$ and $t_{2}$.

Fig. 2(b) shows a cross-flow alternative to the contrapropagation flowmeter arrangement, where the static speed of sound $c$ in $\mathrm{m} / \mathrm{s}$ is measured normal to the flow direction. The fluid velocity $v$ along the ultrasonic path can be calculated using the following relationships modified from Sanderson [7].

$v=\frac{c t_{1} \sin \theta-d}{t_{1} \sin \theta \cos \theta}$

$c=L_{\mathrm{C}} / t_{\mathrm{c}}$

where $t_{\mathrm{c}}$ is the time of flight in seconds for the cross-flow path $L_{C}$. Transit time ultrasonic flowmeters measure the mean fluid velocity on the chord between the transducer faces along the ultrasonic path. The mean fluid flow velocity $v_{\mathrm{m}}$ in the pipeline is related to the velocity $v$ along the ultrasonic path by a meter factor $k$ which is defined as follows [20]:

$k=v / v_{\mathrm{m}}$

The mean pipe velocity may then be multiplied by the pipe cross sectional area to give the volumetric flowrate.

\section{Experimental details}

In order to investigate the application of electrostatic ultrasonic transducers to gas flowmetry, a test rig shown schematically in Fig. 3 was constructed from a $0.1 \mathrm{~m}$ diameter smooth PVC pipe. Interchangeable metering sections consisting of spool piece arrangements as shown in Fig. 2(a) and (b) were manufactured. A centrifugal blower producing air flows of up to $17.5 \mathrm{~m} / \mathrm{s}$ was mounted 20 diameters upstream of the spool piece, and (a)

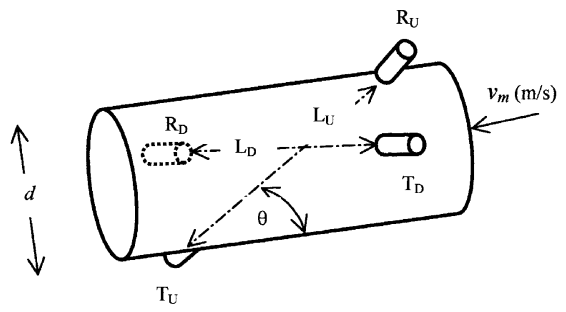

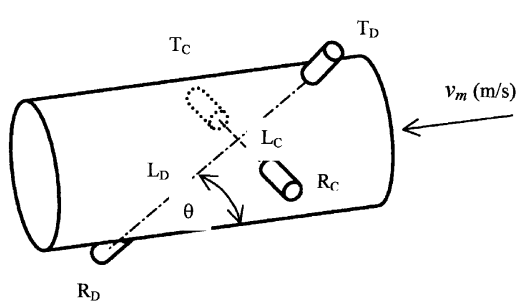

Fig. 2. (a) contra-propagation spool piece arrangement (transducers $90^{\circ}$ out of plane) and (b) cross-flow spool piece arrangement (T: transmitter, R: receiver, $L$ : ultrasonic path length, $d$ : pipe diameter, $v$ : fluid velocity; subscripts, C: crossflow, D: downstream, U: upstream). 


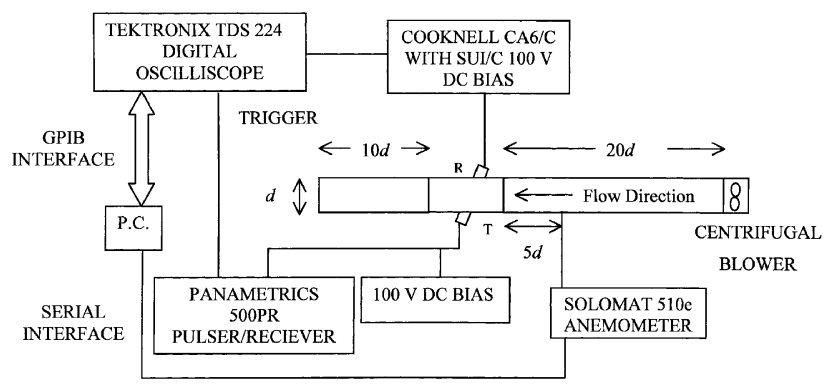

Fig. 3. Experimental set up for time of flight measurement. Note: only one pair of transmit/receive transducers is shown.

the downstream distance to the outlet was 10 diameters to ensure fully developed flow profiles in the metering section. Ultrasound was generated and received by two pairs of electrostatic transducers with $15 \mathrm{~mm}$ apertures and $5 \mu \mathrm{m}$ metallised Mylar film. The transmitters were driven by a Panametrics Pulser/Receiver (Model 500PR) providing a $-250 \mathrm{~V}$ pulse with a pulse energy of up to $19.4 \mu \mathrm{J}$. A Cooknell CA6/C charge amplifier with a sensitivity of $250 \mathrm{mV} / \mathrm{pC}$ amplified each received signal, which was then digitised and displayed on a Tektronix TDS 224 oscilloscope before being transferred to a PC via a GPIB interface for storage and analysis. All transducers had an applied dc bias voltage of $+100 \mathrm{~V}$. The mean pipeline velocity $v_{\mathrm{m}}$ was measured 5 diameters upstream of the metering section using a Solomat 510e anemometer, and was transferred to a PC via an RS232 interface for storage and analysis.

\section{Results and discussion}

The velocity of flow in the test rig was determined at flowrates up to $17.5 \mathrm{~m} / \mathrm{s}$ using both the anemometer and the ultrasonic system. The flow regime for this range of flowrates is turbulent, given that the Reynolds numbers range up to 131250 . Typical ultrasonic waveforms from the contra-propagating arrangement are shown in Fig. 4(a) which shows the signals obtained with no flow in the pipe and Fig. 4(b) which shows the changes in propagation times produced by a flow of $5 \mathrm{~m} / \mathrm{s}$. The signals are well damped at a centre frequency of 0.6 $\mathrm{MHz}$ with a $-6 \mathrm{~dB}$ bandwidth of $0.5 \mathrm{MHz}$. Similar waveforms were obtained from the cross-flow spool piece. Fig. 5 shows frequency spectra from the upstream path of the contra-propagating spool piece at 0,5 and 11 $\mathrm{m} / \mathrm{s}$ showing that a flow-dependent frequency shift may be measured simultaneously with the transit time signals.

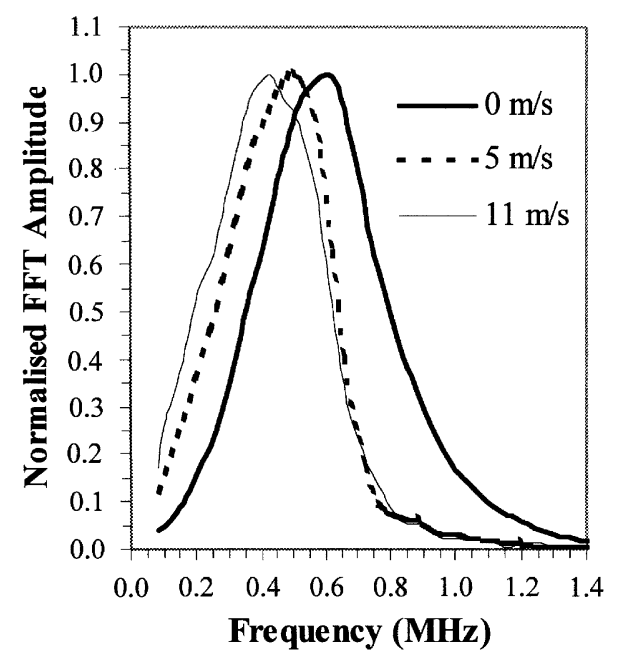

Fig. 5. Shift in frequency with flowrate of waveforms from the upstream path in the contra-propagating spool piece. (a)

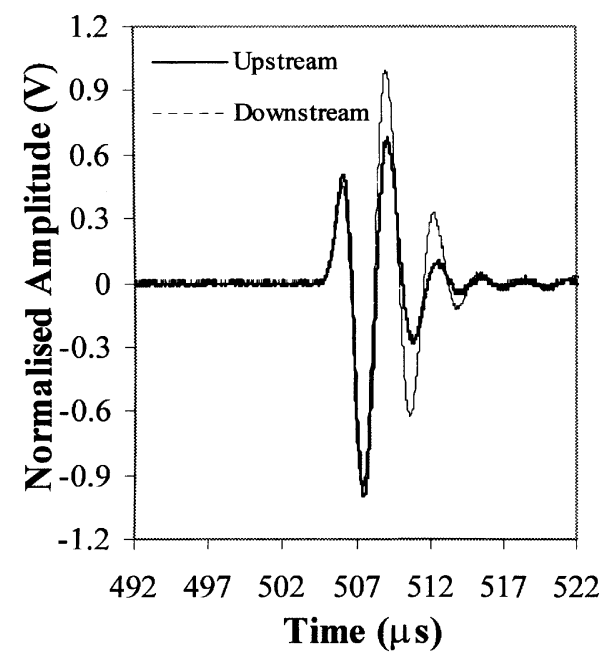

(b)

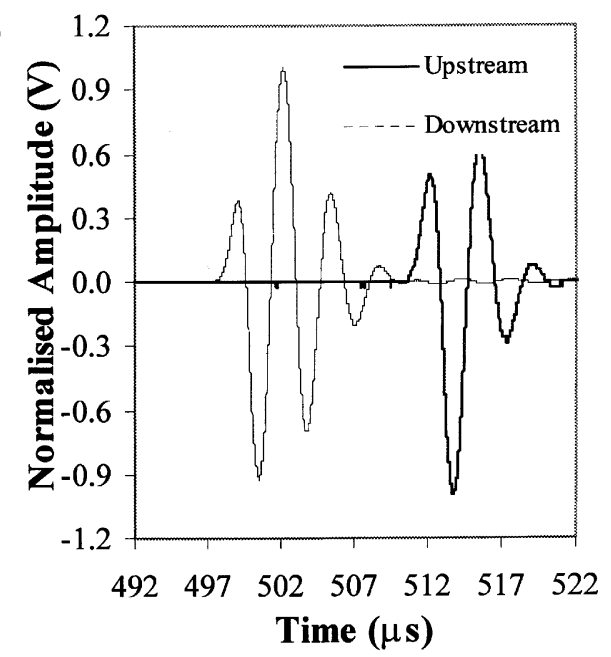

Fig. 4. (a) Ultrasonic waveforms from the contra-propagation spool piece at zero flow. (b) Ultrasonic waveforms from the contra-propagation spool piece at $5 \mathrm{~m} / \mathrm{s}$. 


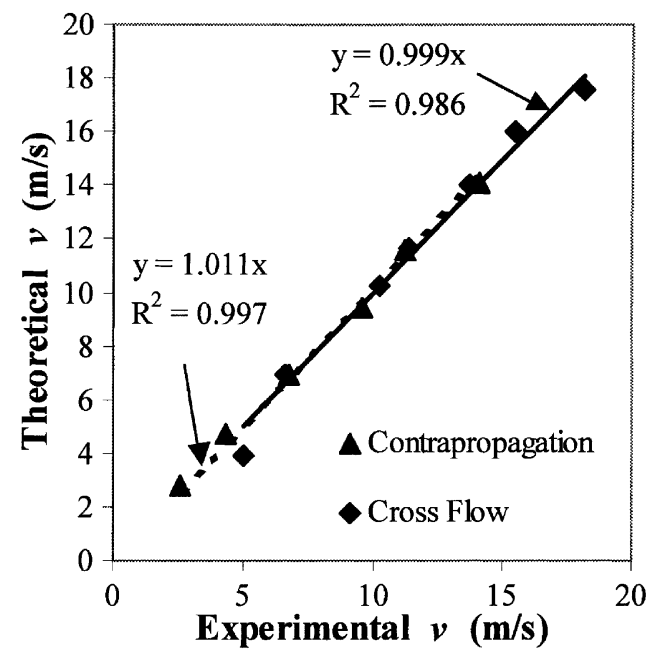

Fig. 6. Experimental vs Numerical values of flow velocity, $v$.
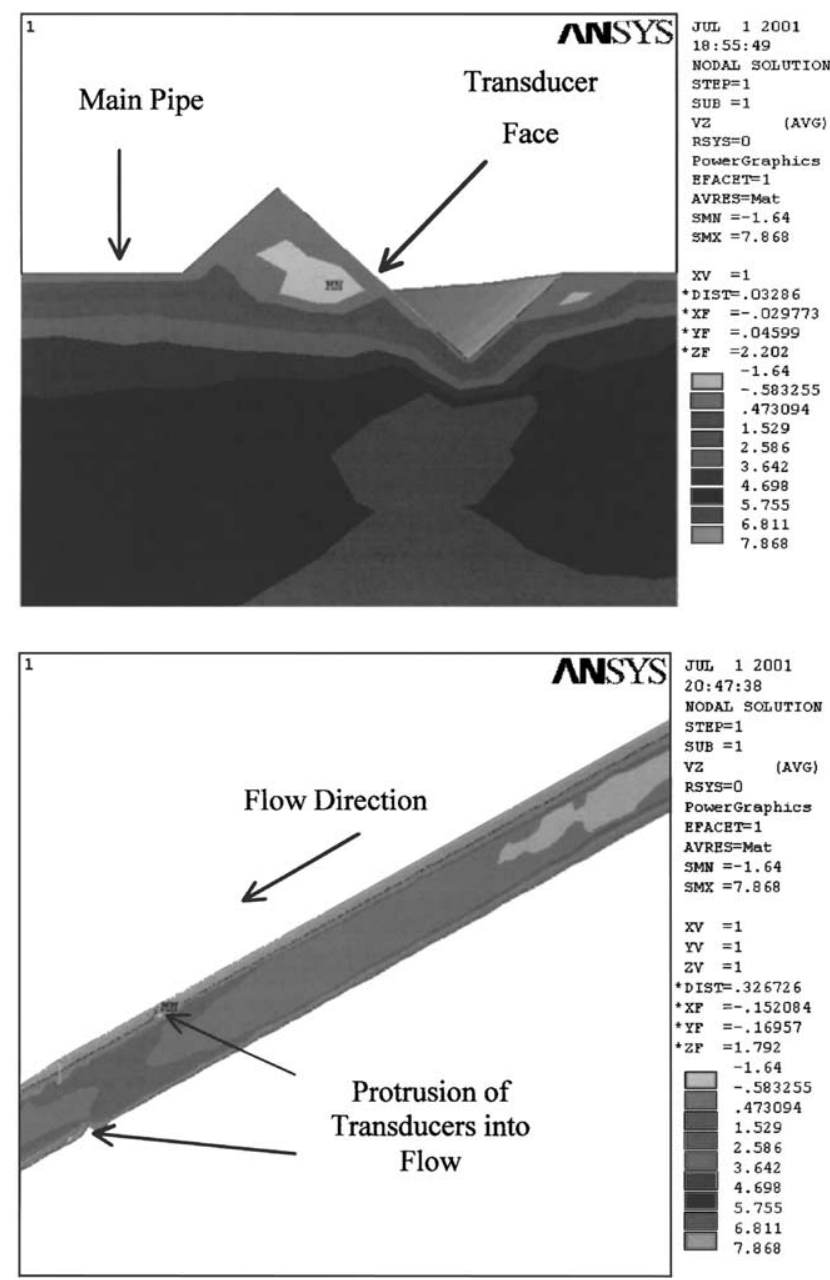

Fig. 7. (a) FLOTRAN flow velocity nodal solution of transducer port showing protrusion into main flow. (b) Typical FLOTRAN flow velocity nodal solution.

\section{Theoretical modelling}

Experimental $k$ factor values of 0.9185 and 0.9213 were determined for the cross-flow and contra-propagating flowmeters respectively. Both spool piece arrangements were modelled in FLOTRAN to determine theoretical values of $v$ at various values of $v_{\mathrm{m}}$. These results were subsequently compared with experimental values of $v$ determined ultrasonically at the same $v_{\mathrm{m}}$ values. As shown in Fig. 6, regression analysis indicated excellent correlation between the experimental and theoretical data, with $R^{2}$ values of 0.997 and 0.986 for the contra-propagation and cross-flow data respectively. Fig. 7(a) shows a typical FLOTRAN velocity nodal solution of a transducer port when $v_{\mathrm{m}}$ is set at $5 \mathrm{~m} / \mathrm{s}$. Fig. 7(b) shows a typical FLOTRAN velocity nodal solution when $v_{\mathrm{m}}$ has a value of $5 \mathrm{~m} / \mathrm{s}$, with a well-developed flow profile upstream of the metering section and minimum flow disruption along the ultrasonic path, but a slight reduction in flow velocity in the metering section.

\section{Conclusions}

The research has shown that electrostatic ultrasonic transducers are suitable for gas flow metering applications. Contra-propagating and cross-flow transducer spool piece configurations were investigated, and determined flowrates up to $17.5 \mathrm{~m} / \mathrm{s}$ with a great degree of accuracy. The values of $v$ determined ultrasonically using both spool pieces were in excellent agreement with the numerical FLOTRAN simulations. A flow-dependent shift in frequency was observed simultaneously with the transit-time measurements.

\section{References}

[1] A.T.J. Hayward, Flowmeters: A Basic Guide and Source-book for Users, Macmillan, London, 1979.

[2] J. Yoder, Raising the bar for the primary elements, Control Engineering 47 (11) (2000) 65-68.

[3] S.A. Sherif, R.H. Pletcher, Analytical procedures for predicting the response of constant-temperature hot-wire and film anemometers, Journal of International Measurement Federation 25 (3) (1999) 193-201.

[4] P.M. Downing, Modern vane anemometers and their use for fume hood monitoring, ASHRAE Transactions, Proceedings of the 1997 Meeting 103 (1) (1997) 223-227.

[5] R.C. Baker, Flow Measurement, Mechanical Engineering Publications, London, 1989.

[6] H. Dane, Ultrasonic Measurment of Unsteady Gas Flow, Flow Measurement and Instrumentation 8 (3-4) (1997) 183-190.

[7] M.L. Sanderson, Industrial Flow Measurement by Ultrasonics, Insight 41 (1) (1999) 16-19.

[8] G. Vass, Ultrasonic Flowmeter Basics, Sensors 14 (10) (1997) $73-78$.

[9] L. Lynnworth, Ultrasonic Measurements for Process Control, Academic Press, London, 1989. 
[10] Y. Liu, L.C. Lynnworth, T.H. Nguyen, D. Xiao, J. Walters, Ultrasonic transducers for measuring air flow near one bar and high temperature fluid flows up to 100 bar, Proceedings of the International Pipeline Conference. 2 (1996) 1055-1062.

[11] A. Gachagan, G. Hayward, S.P. Kelly, W. Galbraith, Characterization of air-coupled transducers, IEEE Transactions on Ultrasonics, Ferroelectrics, and Frequency Control 43 (4) (1996) 678-689.

[12] C. Wykes, H. Carr, Diagnostic measurements in capacitive transducers, Ultrasonics 31 (1) (1993) 13-20.

[13] P. Mattila, J. Stor-Pellinen, J. Ignatius, J. Hietanen, M. Luukkala, Capacitive ultrasonic transducer with net backplate, Measurement Science and Technology 11 (11) (1998) 1119-1125.

[14] D.W. Schindel, D.A. Hutchins, L. Zou, M. Sayer, The design and characterization of micromachined air-coupled capacitance transducers, IEEE Transactions, Ultrasonics, Ferroelectrics, Frequency Control 42 (1995) 42-50.

[15] B.T. Khuri-Yakub, C. Cheng, F. Degertekin, S. Ergun, S. Hansen, $X$. Jin, O. Oralkan, Silicon micromachined ultrasonic transducers, Japanese Journal of Applied Physics, Part 1: Regular
Papers and Short Notes and Review Papers 39 (5) (2000) 2883-2887.

[16] W.M.D. Wright, D.A. Hutchins, D.W. Schindel, P.W. Carpenter, D.P. Jansen, Ultrasonic tomographic imaging of temperature and flow fields in gases using air-coupled capacitance transducers, J. Acoust. Soc. Am. 104 ( 6) (1998) 3446-3455.

[17] M. Calzolai, L. Capineri, A. Fort, L. Masotti, S. Rocchi, M Scabia, A 3-D PW ultrasonic Doppler Flowmeter: Theory and Experimental Characterization, IEEE Transactions on Ultrasonics Ferroelectrics and Frequency Control 46 (1) (1999) 108-113.

[18] A. Worch, Clamp-on ultrasonic cross-correlation flowmeter for one phase flow, Measurement Science and Technology 9 (4) (1998) 622-630.

[19] V. Hans, G. Poppen, E. vonLavante, S. Perpeet, Vortex shedding flowmeters and ultrasound detection: signal processing and influence of bluff body geometry, Flow measurement and Instrumentation 9 (2) (1998) 79-82.

[20] G. de Boer, Ultrasonic gas flow measurement for custody transfer applications, Proceedings of the International Pipeline Conference 2 (1996) 1063-1075. 